\title{
The homogeneous mutation status of a 22 gene panel justifies the use of serial sections of colorectal cancer tissue for external quality assessment
}

\author{
Jeroen R. Dijkstra ${ }^{1}$ - Bastiaan B. J. Tops ${ }^{1}$ - Iris D. Nagtegaal ${ }^{1}$ • J. Han J. M. van \\ Krieken $^{1} \cdot$ Marjolijn J. L. Ligtenberg ${ }^{1,2}$
}

Received: 29 December 2014 / Accepted: 13 May 2015 / Published online: 6 June 2015

(C) The Author(s) 2015. This article is published with open access at Springerlink.com

\begin{abstract}
Testing for treatment related biomarkers in clinical care, like Ras mutation status in colorectal cancer (CRC), has increased drastically over recent years. Reliable testing of these markers is pivotal for optimal treatment of patients. Participation in external quality assessment (EQA) programs is an important element in quality management and often obligatory to comply with regulations or for accreditation. Formalin-fixed paraffin-embedded (FFPE) clinical specimens would ideally form the basis for these assessments, as they represent the most common starting material for molecular testing. However, molecular heterogeneity of a lesion in a FFPE tissue block could potentially affect test results of participating laboratories, which might compromise reliability of the quality assessment results. To assess the actual impact of this potential problem, we determined the mutation status of 22 genes commonly mutated in colon cancer in four levels covering $360 \mu \mathrm{m}$ of 30 FFPE tissue blocks, by Next Generation Sequencing. In each block, the genotype of these genes was identical at all four levels, with only little variation in mutation load. This result shows that the mutation status of the selected 22 genes in CRC specimens is homogeneous within a $360 \mu \mathrm{m}$ segment of the tumor. These data justify the use of serial sections, within a defined segment of a
\end{abstract}

Electronic supplementary material The online version of this article (doi:10.1007/s00428-015-1789-5) contains supplementary material, which is available to authorized users.

Jeroen R. Dijkstra

Jeroen.Dijkstra@radboudumc.nl

1 Department of Pathology, Radboud University Medical Center, PO Box 9101, 6500, HB Nijmegen, The Netherlands

2 Department of Human Genetics, Radboud University Medical Center, Nijmegen, The Netherlands
CRC tissue block, for external quality assessment of mutation analysis.

Keywords Colon $\cdot$ RAS $\cdot$ Reproducibility $\cdot$ Mutant allele frequency

\section{Introduction}

In recent years, testing of tumor-specific molecular biomarkers to predict the putative response to targeted therapeutics has increased rapidly. An example is the incorporation in standard care of the mutation status of KRAS and NRAS, which is decisive in starting epidermal growth factor receptor (EGFR)-targeted therapy on metastatic colorectal cancer patients. The European Medicines Agency (EMA) declared that the use of EGFR-targeted therapies should be restricted to patients with a Ras wild-type tumor [1,2]. Mutations in other genes, such as BRAF and PIK3CA, are now tested in the context of clinical trials and may enter clinical practice in the near future.

Quality assessment is essential to validate the results of these molecular tests, in order to achieve or sustain optimal patient treatment [3]. Furthermore, quality assessment allows comparison of inter-laboratory results, which is pivotal to determine the effectiveness of a treatment regime instigated by the result of a molecular test. To assure high quality of testing, it is essential that each laboratory properly validates its workflow of molecular tests, typically a sequence of techniques [4-7] which for mutation screening consists of DNA extraction, PCR amplification, sequencing, and detection. Test results need to be validated, and participation in external quality assessment (EQA) programs is often obligatory to comply with regulations or a requirement for accreditation as a test provider $[8,9]$. 
Formalin-fixed paraffin-embedded (FFPE) tissue is the most common starting material for molecular testing, independent of the exact workflow or test origin (laboratory developed test or commercially available). Therefore, they provide the ideal basis for validation, EQA programs, and for comparison of method performance. Reference samples need to yield highly reproducible results over the participating laboratories. This is easy to attain with homogeneous liquid samples from which random samples can be drawn but potentially problematic for serial tissue sections from an intrinsically heterogeneous tissue block. Reproducibility problems can arise due to a variety of factors, including variation in the surface area occupied by tumor tissue, proportion of neoplastic cells relative to stromal cells in tumor tissue and/or heterogeneity of molecular abnormalities within a given block of tumor tissue.

Next generation sequencing (NGS) based techniques are rapidly becoming the new standard to evaluate the mutation status of a sample of tumor tissue. They provide highly sensitive semi-quantitative information on all possible mutations in a region of interest of the genome. To assess mutation status of the most commonly mutated genes in colon cancer, a colonlung cancer panel was designed that allows reliable mutation analysis (on only $10 \mathrm{ng}$ of DNA) of the most frequently mutated regions of the genes for receptor tyrosine kinases $E G F R$, ERBB2, ERBB 4, MET, ALK, FGFR1, FGFR2, FGFR3, and $D D R 2$, their pathway genes KRAS, NRAS, BRAF, PIK3CA, and MAP2K1, as well as TP53, STK11, CTNNB1, SMAD4, FBXW7, and NOTCH1, on only 10 ng of DNA [10].

We evaluated the reproducibility of genotyping for this panel of tissue sections at different depths of CRC tissue blocks, as this is important for the design of EQA schemes evaluating gene testing for RAS and eventually other genes in the panel, in view of the requirement that serial sections at different depths of a tumor block should generate the same results.

\section{Materials and methods}

\section{Samples}

FFPE tissue samples from resected primary colorectal cancer specimens, stage T3N0M0 or higher and with at least $40 \%$ of neoplastic cells, were obtained from 30 patients who had been treated at the Radboud University Medical Center (RadboudUMC) between 2007 and 2013. Six tumors were microsatellite instable (MSI), 24 microsatellite stable (MSS), and 8 were from patients treated with neoadjuvant chemotherapy. The samples were anonymized for quality assessment purposes, as a result of which they were not subjected to research regulations of RadboudUMC, which require informed consent and permission by the internal review board.

\section{DNA extraction}

FFPE blocks were cut to obtain 63 consecutive sections of $6 \mu \mathrm{m}$. H\&E staining was performed on sections 1, 20, 40, and 60. A pathologist assessed each stained section individually and selected the region for macrodissection and estimated the percentage of neoplastic cells. Macrodissected tumor material from two sections following each H\&E stained section was pooled for DNA extraction (Fig. 1). Tissue was digested in $110 \mu \mathrm{l} 5 \%$ Chelex-100 resin (Bio-Rad laboratories, Hercules, CA) and $2 \mathrm{mg} / \mathrm{ml}$ proteinase $\mathrm{K}$ in $10 \mathrm{mM}$ Tris/ $\mathrm{HCl}(\mathrm{pH} 8.5), 1 \mathrm{mM}$ EDTA (pH 8.0), $0.01 \%$ Tween20. Following overnight incubation at $56{ }^{\circ} \mathrm{C}$, the enzyme was heat inactivated at $95{ }^{\circ} \mathrm{C}$ for $10 \mathrm{~min}$. After two rounds of centrifugation $(16,000 \mathrm{rpm}$ for $1 \mathrm{~min})$ the DNA in the supernatant was quantified using the Qubit platform (Life Technologies, Foster City, CA).

\section{Sequencing}

Bar-coded libraries were prepared from 10 ng DNA using the Ion AmpliSeq Colon and Lung Cancer Panel version 1 (Life Technologies), essentially as described by the manufacturer except for amplification with 23 instead of 21 cycles. Clonal amplification was subsequently performed by emulsion PCR using the One Touch 2 system (Life Technologies). DNA concentration of the resulting libraries was measured using the Qubit platform (Life Technologies). Libraries were quantitatively pooled and run on the Ion Torrent Personal Genome Machine (Life Technologies). Torrent Suite Software v.3.4.2 was used to pre-process raw data. Subsequently, sequence alignment and variant calling were performed using SeqNext

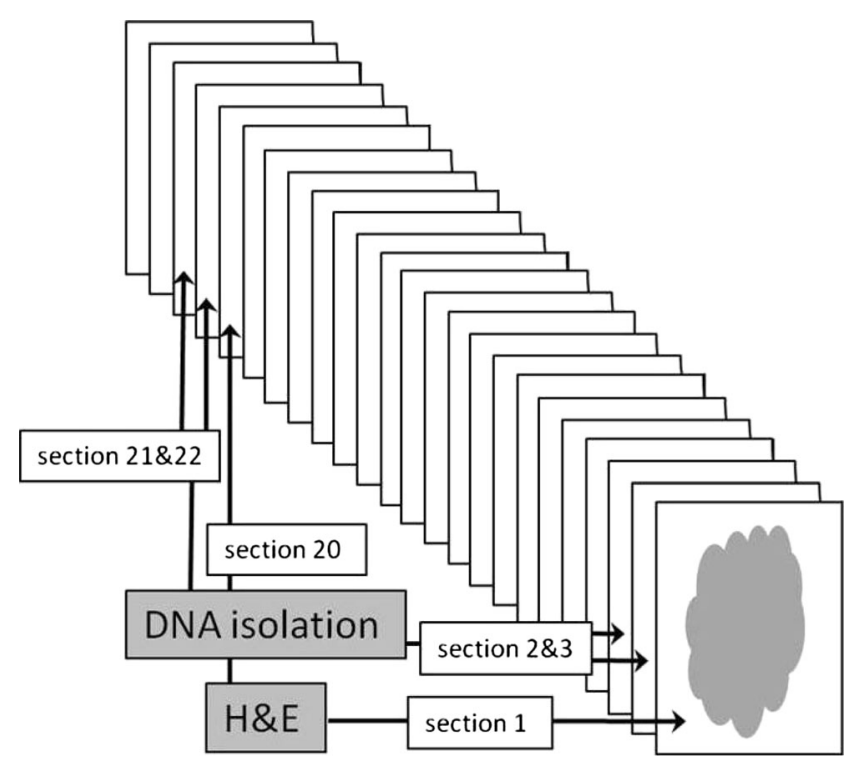

Fig. 1 Schematic display of the use of the designated sections. All sections had a thickness of $6 \mu \mathrm{m}$ 
software v.4.1.2 (JSI medical systems GmbH, Kippenheim, Germany). Each nucleotide was covered with a minimum of at least 50 reads, and variants were included if they were found at an allelic frequency of at least $10 \%$.

\section{Data analysis}

Variants were filtered for known SNPs (MAF $\geq 0.02)$, synonymous mutations, and variants most likely to be false positive. The latter were defined as variants present in most/all of the 120 DNA samples. The remaining mutations were assessed in the Catalogue of Somatic Mutations in Cancer (COSMIC) database [11]. Variants not present in the COSMIC database were subjected to Sanger sequencing to confirm the somatic origin of the specific variant (in normal and tumor tissue).

Differences between the percentages of mutated reads of individual mutations at different levels of a tissue block were evaluated by ANOVA, assuming repeated measurements.

\section{Results}

To assess reproducibility of genotyping within one FFPE colorectal tumor tissue block, 63 sections of $6 \mu \mathrm{m}$ were cut from 30 different tumors. Sections 1 (level A), 20 (level B), 40 (level C), and 60 (level D) were histologically evaluated (Fig. 1). Of each sample, a comparable region was macrodissected at each level. The estimated percentage of neoplastic cells was similar for the different levels within a block with a maximum of $20 \%$ difference from level A (mean $3.56 \%$, st dev 5.67) (supplementary data).

Mutation analysis using the Ion AmpliSeq Colon and Lung Cancer Panel version 1 (Life Technologies), was performed on DNA isolated from the macrodissected region of sections 2 and 3 (level A), sections 21, and 22 (level B), sections 41, and 42 (level C) and sections 61 and 62 (level D). Because of poor quality of the DNA from the initial isolation of tumor 18 sections 23 and 24, sections 43 and 44, and section 63 and 64 were used as levels B, C, and D, respectively. Variant calling in $K R A S$ (exon 2, 3, and 4), NRAS (exon 2 and 3), $B R A F$ (exon 15), and PIK3CA (exon 10 and 21) was
Table 2 Mean percentage of mutated reads per gene

\begin{tabular}{llllll}
\hline & & \multicolumn{4}{l}{ Average \% mutated reads (st. dev) } \\
\cline { 3 - 6 } Gene & $n$ & Level A & Level B & Level C & Level D \\
\hline BRAF & 6 & $39(22)$ & $38(16)$ & $38(14)$ & $39(13)$ \\
DDR2 & 1 & 30 & 40 & 39 & 32 \\
ERBB4 & 1 & 32 & 31 & 39 & 39 \\
FBXW7 & 4 & $36(7)$ & $31(4)$ & $35(7)$ & $33(3)$ \\
FGFR3 & 1 & 38 & 27 & 25 & 30 \\
KRAS & 9 & $37(12)$ & $36(9)$ & $39(14)$ & $35(12)$ \\
MAP2K1 & 1 & 24 & 34 & 33 & 21 \\
NRAS & 1 & 34 & 39 & 39 & 43 \\
PIK3CA & 5 & $26(4)$ & $25(6)$ & $27(4)$ & $26(6)$ \\
SMAD4 & 4 & $31(9)$ & $31(12)$ & $32(8)$ & $36(7)$ \\
TP53 & 17 & $47(17)$ & $43(15)$ & $45(16)$ & $47(16)$ \\
\hline
\end{tabular}

performed on nucleotide coverage of at least 160 reads. The overall lowest mean coverage for these exons was 2396, with a standard deviation of 1449 (Table 1).

The number of somatic mutations detected in the different tumors varied from zero to five with a mean of 3.0 in microsatellite instable, 1.4 in microsatellite stable tumors of patients not subjected to neoadjuvant therapy and 1.4 in microsatellite stable tumors of patients subjected to neo-adjuvant therapy (supplementary data). Mutations in KRAS, NRAS, BRAF, and $P I K 3 C A$ were detected in $9,1,6$, and 5 tumors, respectively (Table 2). The most frequently mutated gene was TP53, mutated in 17 tumors. For all mutations the concordance detected at levels A, B, C, and D was $100 \%$.

The percentage of variant reads was used to assess the variation in mutation load at the different levels within each tumor block. This varied between different mutations within a given tumor (Fig. 2). However, for a specific mutation the mutation load was stable at all four levels (Fig. 3), as there were no significant differences in percentage of mutant reads between levels A, B, C, and D $(p=0.392)$. This indicates that NGS is highly reproducible and that heterogeneity between sections at four different levels of tissue blocks is limited.
Table 1 Coverage data of individual fragments of $K R A S$, $N R A S, B R A F$, and PIK3CA

\begin{tabular}{|c|c|c|c|c|c|c|c|c|}
\hline & \multicolumn{3}{|l|}{$K R A S$} & \multirow{2}{*}{$\begin{array}{l}B R A F \\
\text { Exon } 15\end{array}$} & \multicolumn{2}{|l|}{$N R A S$} & \multicolumn{2}{|l|}{ PIK3CA } \\
\hline & Exon 2 & Exon 3 & Exon 4 & & Exon 2 & Exon 3 & Exon 10 & Exon 21 \\
\hline Min & 186 & 278 & 256 & 175 & 162 & 160 & 233 & 194 \\
\hline Average & 3009 & 4990 & 3304 & 2740 & 3572 & 3783 & 4597 & 3457 \\
\hline St. dev & 1505 & 1963 & 1750 & 1449 & 1395 & 1396 & 1585 & 1376 \\
\hline
\end{tabular}


MSI

MSS
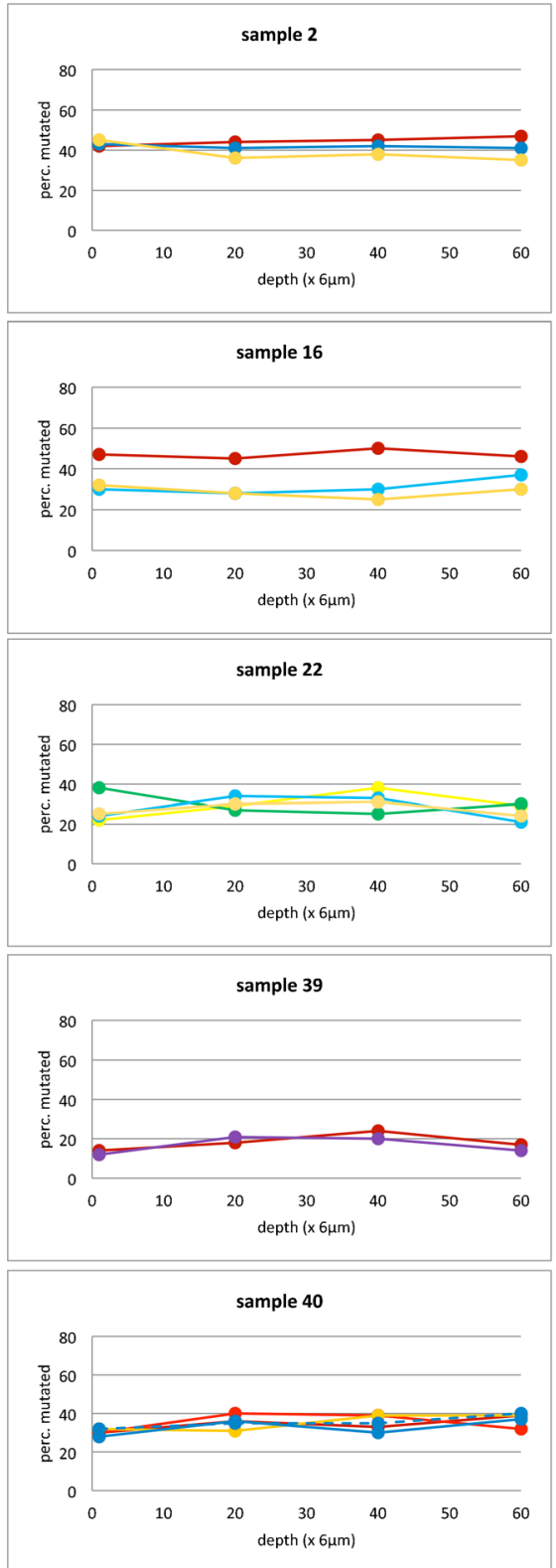

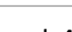

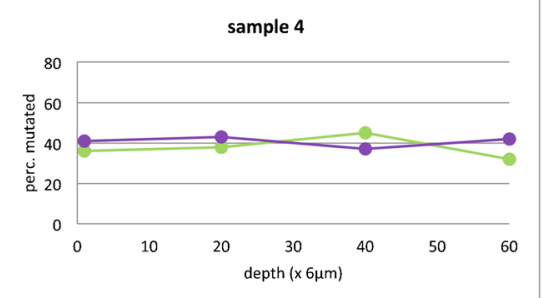

sample 8

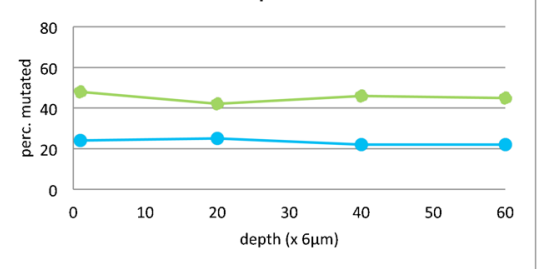

sample 9
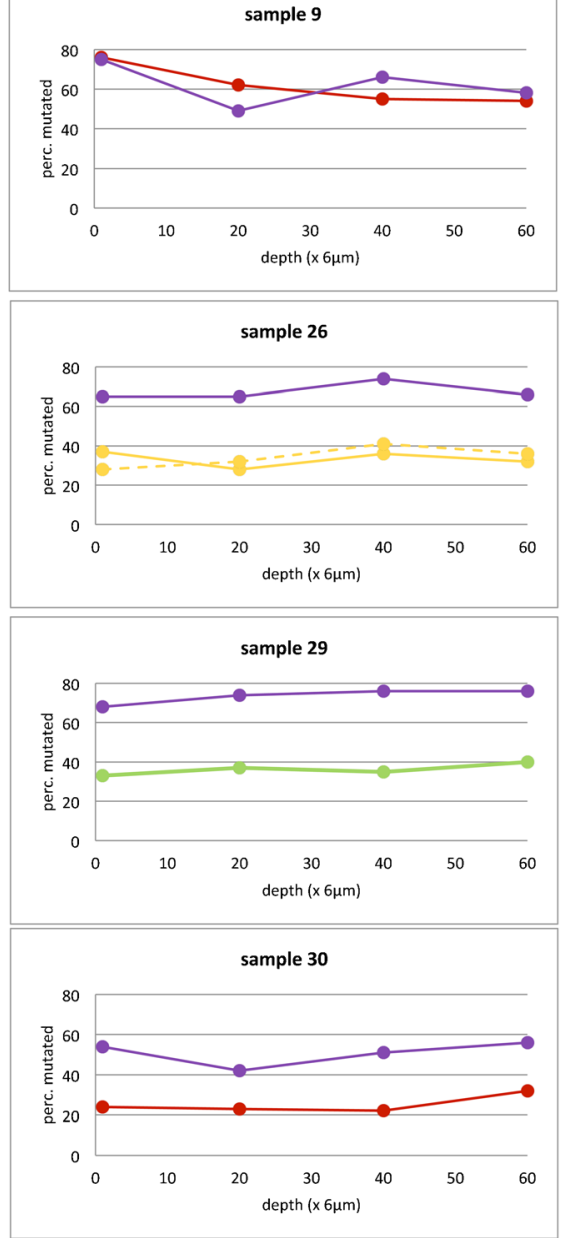

MSS + neoadjuvante chemotherapy
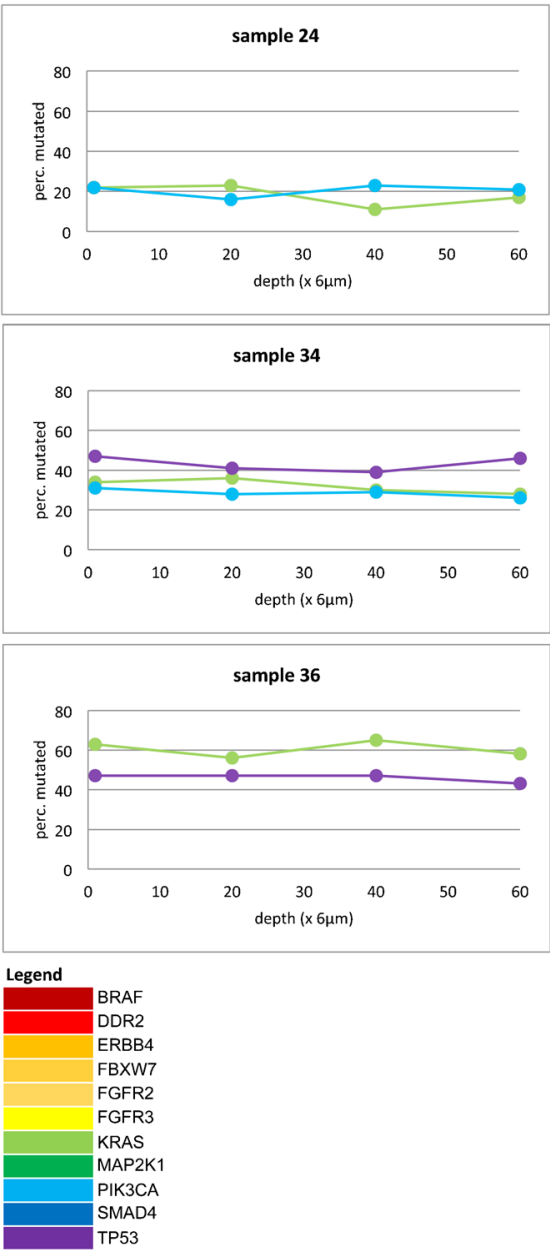

Fig. 2 Percentage of mutated reads per mutation for each sample that contains multiple mutations at different levels of the FFPE tissue block (depth). Mutations are represented as color-coded (per gene) continuous lines, except for sample 26 and 40 since in these samples more than one mutation per gene was found 


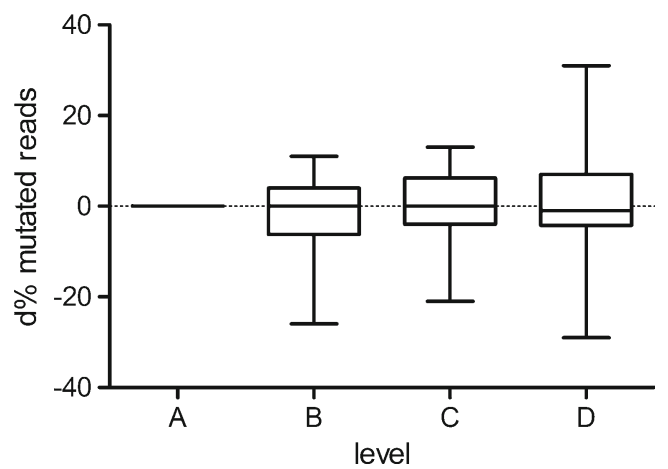

Fig. 3 Difference in percentage of mutated reads of all individual mutations at levels B, C, and D in comparison to level A. Difference in percentage of mutant reads at levels B, C, and D compared to that of level A: $p=0.392$, repeated measures ANOVA

\section{Discussion}

For each of 30 colorectal tumor blocks tested at four levels separated by $120 \mu \mathrm{m}$ (hence, a segment of $360 \mu \mathrm{m}$ between the upper and lower level), the genotype of 22 genes analyzed by next generation sequencing was the same at all four levels. Between different levels of a given tumor, little variation in the percentage of mutant alleles was observed. This shows that NGS-based genetic testing is reproducible and that different levels in a single block of tumor tissue are homogeneous. The results of these analyses justify the use of sections derived from different levels of the same tumor block in current external quality assessment schemes.

For this study, we selected samples with a relatively high percentage of neoplastic cells to facilitate the detection of mutations present in only a subset of neoplastic cells. The mutation load of different mutations in a given tumor differed by a factor two at most, which might reflect subtle copy number changes or copy number neutral loss of heterozygosity events. In four samples $(22,24,31$, and 39), the percentage of mutant alleles was less than $30 \%$ of the percentage of neoplastic cells, which is clearly lower than would be expected if one allele of a given diploid gene would be mutated in all neoplastic cells. This might be due to overestimation of the percentage of neoplastic cells by the pathologist, copy number gains of wild-type alleles, or the presence of these mutations in only a subset of the neoplastic cells. However, as in two (sample 22 and 39) of the three tumors this applies to mutations in multiple genes residing on different chromosomes, overestimation of the percentage of neoplastic cells is the most likely explanation. Therefore, our results do not strongly indicate the existence of tumor heterogeneity for the tested genes in this setting.

In our study, we concentrated on only 30 tumors that were judged eligible for use in quality assessment schemes. This implied morphological homogeneity and avoidance of precancerous regions that might harbor fewer mutations. Differences in mutation load between morphologically different regions within one slide were not examined. Moreover, we studied regions that were at most $360 \mu \mathrm{m}$ apart, which is far less than the diameter of most tumors. Therefore, our data cannot be used to argue in favor of homogeneity of mutation status throughout an entire primary tumor.

In our next generation sequencing panel, we tested for mutations in genes frequently mutated in colorectal cancer. The panel allows the detection of mutations that are currently considered as actionable (NRAS, KRAS) or putatively actionable (BRAF, PIK3CA) in colorectal cancer. The current design of EQA schemes, which distribute sections from different levels of a tissue block and aim at a sensitivity of $10 \%$ mutant alleles, seems therefore also appropriate for these putatively actionable mutations. For reliable detection of mutant alleles present at a lower percentage, other more sensitive approaches would be necessary. However, the clinical relevance of mutations in a low percentage of neoplastic cells is as yet not clear and therefore is not an aim for current EQA schemes.

In conclusion, our data show that the percentage of mutant alleles, of a panel of 22 genes including those that are frequently mutated in colorectal cancer, is similar within a defined depth of a block of colorectal cancer tissue. This justifies the use of serial sections to assess the quality of mutation analyses in different laboratories, as has become customary in EQA schemes.

Funding This study was funded by Amgen, which did not have any influence on the design and analysis of the results.

Conflict of interest The authors declare that they have no competing interests.

Open Access This article is distributed under the terms of the Creative Commons Attribution 4.0 International License (http:// creativecommons.org/licenses/by/4.0/), which permits unrestricted use, distribution, and reproduction in any medium, provided you give appropriate credit to the original author(s) and the source, provide a link to the Creative Commons license, and indicate if changes were made.

\section{References}

1. EMA (2013) European Medicine Agency. Vectibix: EPAR summary for the public. http://www.ema.europa.eu/docs/en GB/ document_library/EPAR__Summary_for_the_public/human/ 000741/WC 500047704.pdf. Accessed 3 September 2014

2. EMA (2014) European Medicine Agency. Erbitux: EPAR summary for the public. http://www.ema.europa.eu/docs/en_GB/document library/EPAR - Summary for the public/human/000558/ WC500029111.pdf. Accessed 3 September 2014

3. Kalman LV, Lubin IM, Barker S, du Sart D, Elles R, Grody WW, Pazzagli M, Richards S, Schrijver I, Zehnbauer B (2013) Current landscape and new paradigms of proficiency testing and external 
quality assessment for molecular genetics. Arch Pathol Lab Med 137(7):983-988. doi:10.5858/arpa.2012-0311-RA

4. Deans ZC, Bilbe N, O'Sullivan B, Lazarou LP, de Castro DG, Parry S, Dodson A, Taniere P, Clark C, Butler R (2013) Improvement in the quality of molecular analysis of EGFR in non-small-cell lung cancer detected by three rounds of external quality assessment. J Clin Pathol 66(4):319-325. doi:10.1136/jclinpath-2012-201227

5. Emile JF, Tisserand J, Bergougnoux L, Nowak F, Faucher G, Surel S, Lamy A, Lecorre D, Helias-Rodzewicz Z, Hofman P, Sabourin JC, Laurent-Puig P, Group BE (2013) Improvement of the quality of BRAF testing in melanomas with nationwide external quality assessment, for the BRAF EQA group. BMC Cancer 13:472. doi: 10.1186/1471-2407-13-472

6. Cree IA, Deans Z, Ligtenberg MJ, Normanno N, Edsjo A, Rouleau E, Sole F, Thunnissen E, Timens W, Schuuring E, Dequeker E, Murray S, Dietel M, Groenen P, Van Krieken JH, European Society of Pathology Task Force on Quality Assurance in Molecular P, the Royal College of P (2014) Guidance for laboratories performing molecular pathology for cancer patients. J Clin Pathol 67(11):923-931. doi:10.1136/jclinpath-2014-202404

7. Dubbink HJ, Deans ZC, Tops BB, van Kemenade FJ, Koljenovic S, van Krieken HJ, Blokx WA, Dinjens WN, Groenen PJ (2014) Next generation diagnostic molecular pathology: critical appraisal of quality assurance in Europe. Mol Oncol 8(4):830-839. doi:10. 1016/j.molonc.2014.03.004

8. ISO (2007) International Organization for Standardization, ISO 15189 medical laboratories - particular requirements for quality and competence

9. Nowak F, Calvo F, Soria JC (2013) Europe does it better: molecular testing across a national health care system-the French example. Am Soc Clin Oncol educ Book / ASCO Am Soc Clin Oncol Meeting:332-337. doi:10.1200/EdBook_AM.2013.33.332

10. Tops B, Normanno N, Kurth H, Amato E, Mafficini A, Rieber N, Le Corre D, Rachiglio A, Reiman A, Sheils O, Noppen C, Lacroix L, Cree IA, Scarpa A, Ligtenberg M, Laurent-Puig P (2015) Development of a semi-conductor sequencing-based panel for genotyping of colon and lung cancer by the Onconetwork consortium. BMC Cancer 15(1):26. doi:10. 1186/s12885-015-1015-5

11. Bamford S, Dawson E, Forbes S, Clements J, Pettett R, Dogan A, Flanagan A, Teague J, Futreal PA, Stratton MR, Wooster R (2004) The COSMIC (Catalogue of Somatic Mutations in Cancer) database and website. Br J Cancer 91(2):355-358. doi:10.1038/sj.bjc. 6601894 